\title{
Dynamics of the defect-mediated desorption of alkali halide surfaces
}

\author{
M. Szymonski, A. Droba, P. Struski, and F. Krok \\ Research Centre for Nanometer-Scale Science and Advanced Materials (NANOSAM) \\ Faculty of Physics, Astronomy and Applied Computer Science, Jagiellonian University \\ 4 Reymonta Str., Krakow 30-059, Poland \\ E-mail: ufszymon@cyf-kr.edu.pl
}

Received April 23, 2012

\begin{abstract}
Dynamic processes leading to desorption of $\mathrm{Rb}$ and I atoms from the RbI (100) surface co-irradiated with $1 \mathrm{keV}$ electrons and visible light (with a wavelength corresponding to the F-center absorption band) have been studied by means of mass-selected time-of-flight (TOF) spectroscopy. Depending on the sample temperature, substantial enhancement of the desorption yield, as well as pronounced changes in the TOF spectra of the emitted atoms have been found. The TOF spectra of halogen atoms consist of two components: thermal (which can be fitted with Maxwellian distribution) and non-thermal one. The non-thermal peak is temperature-independent. There is no non-thermal component for alkali atoms. The comparison of TOF spectra for I atoms emitted from electron bombarded sample with and without simultaneous light irradiation indicates that the yield increase is caused by thermally desorbed atoms, while the non-thermal peak remains unchanged. Presented results confirm well the predictions of the theoretical model of desorption proposed earlier, known as the defect-mediated (F and $\mathrm{H}$ center) desorption of alkali halides.
\end{abstract}

PACS: $62.72 . J-$ Point defects and defect clusters;

68.35.Dv Composition, segregation; defects and impurities;

68.43.-h Chemisorption/physisorption: adsorbates on surfaces.

Keywords: electron, stimulated desorption, alkali halide surtaces, mass-selected spectroscopy.

\section{Introduction}

Electron stimulated desorption (ESD) of alkali halides has been studied intensively for several decades as a model example of inelastic process converting local electronic excitations into a nuclear motion of the sample constituents, and leading to emission of atomic and molecular particles from the eroded surface [1-3]. Similar type of desorption processes caused by electronic transitions has been found in another class of insulating materials, i.e., frozen rare gas solids [4,5]. More recently, such desorption processes have been applied for controlled nanostructuring of wide band gap insulators with the aim of fabricating templates for depositing metal and/or molecular structures fully decoupled electronically from the supporting substrate [6]. In fact, alkali halides nanostructured by electrons have already been used as templates for metal clusters, as well as complex molecule deposition and immobilization [7-9]. Such structures could be applied in molecular electronics.

Kinetic energy of the incident beam of electrons is lost in the alkali halide crystal for electronic excitations, such as electron-hole pairs, and/or surface excitons. Such prima- ry excitations lead to production of so called "Frenkel defects", i.e., F-center and H-center pairs (F-center is an electron trapped in halogen vacancy, while the H-center is an interstitial halogen atom), in the crystal bulk and at the surface. H-center is a very mobile defect, which, activated by the crystal thermal energy, diffuses to the surface leading to emission of halogen atoms. The F-center, when produced in its ground state, cannot efficiently diffuse until activated to the excited state by interaction with an impinging electron, or absorption of light. Arriving at the surface, F-center could neutralize surface alkali ion, and cause its desorption. Whereas the first process, recombination of the $\mathrm{H}$-center with the surface, can cause desorption from any surface site, the recombination of the F-center is more site selective. Puchin et al. [10] have performed APES calculations, that show that only an excited F-center recombining at low-coordinated surface site (such as terrace edge, kink or corner) can cause desorption of alkali atom.

The study of time dependence of the yield of emitted particles, and the atomic force microscopy (AFM) measurements have demonstrated that electron-stimulated de- 
sorption from alkali halide surfaces proceeds in a layer-bylayer mode by growth and linking of pits (of monoatomic depth) on the (100) crystal surface $[11,12]$. It was also demonstrated, that the flux of emitted atoms is anticorrelated with the concentration of F-centers at the surface, or in the subsurface region. Similar process has been observed for the desorption stimulated by UV photons $[13,14]$ and by low energy ions [15]. It has been found, that simultaneous irradiation of the surface with visible light leads to increase of the desorption coefficient, although photons of such energies are unable to cause desorption. They are, however, able to excite the immobile Fcenters trapped in bulk or accumulated on the surface [16], and in this way increase the emission of both alkali atoms (desorbed due to F-centers neutralization) and halogen atoms (since the F-centers in ground state act as traps for diffusing H-centers).

Due to recent advancements of the atomic force microscopy, in particular its dynamic version of frequency modulated AFM performed in an ultrahigh vacuum environment, investigations of the interactions of electrons and ions with crystal surfaces have attracted a renewed interest. Using the AFM technique, it was for the first time possible to study not only what already left the surface but to examine the atomic structure of the crystal right after the desorption process without exposing it to the reactive ambient environment $[11,17]$. This atomistic approach to processes leading to surface modification allowed for establishing a consistent theoretical model describing both the particle emission from bombarded alkali halides, and the surface topography changes due to beam-induced electronic excitation/ionisation $[9,18]$. Finally, it was found that a visible light illumination within the absorption band of the F-centers in desorbed alkali halides could considerably increase the yield of the UV photon stimulated desorption [19]. Since most of the experimental observations, reported so far, yielded qualitatively similar results for both photon- (PSD) and electron- (ESD) stimulated desorption, one would expect that the yield enhancement due to visible light co-excitation should occur for ESD as well as it does for PSD. In fact, such an effect has been found by our group [20] but the results of more systematic measurements have not been reported yet. Therefore, we use this unique occasion of publishing the special Festschrift issue of the Journal "Fizika Nizkikh Temperatur", dedicated to honor Professor Elena V. Savchenko, to contribute with a concluding report on the topic being so close to Elena's scientific interest over the years. In this paper, we report on the first velocity distribution measurements of halogen and alkali atoms desorbed from RbI (100) surface due to ESD with and without co-excitation by visible photon flux. We demonstrate that the thermal component of ESD emitted alkali and halogen atoms is mediated by the excitation and diffusion of ground-state F-centers created during surface irradiation with electron beam leading to an increase of the desorption coefficient for both elements.

\section{Experimental}

The RbI crystals used in our experiment are cleaved in air, and subsequently placed in a vacuum chamber. The pressure in the system is lower than $1 \cdot 10^{-8}$ mbar. The sample is then heated to a $650 \mathrm{~K}$ and annealed in vacuum for several hours to ensure that the surface is free from any contaminations. It has been shown by many previous experiments that such treatment is sufficient for obtaining a clean, well defined (100) surface, with almost no defects (evaporation of low-coordination number atoms is more probable, so the surface defects are removed) [12]. The crystal is irradiated with a $1 \mathrm{keV}$ electron beam, typically at about 5 to $6 \mu \mathrm{A}$ per $\mathrm{cm}^{2}$, i.e., of $4 \cdot 10^{13}$ electrons $/ \mathrm{s}$ per $\mathrm{cm}^{2}$. The emitted $\mathrm{Rb}$ and I atoms are detected and analyzed by the quadrupole mass spectrometer equipped with an electron impact ioniser. The sample temperature is varied in the range $330-390 \mathrm{~K}$ as measured by a thermocouple mounted to the sample holder. As a source of visible light, a conventional halogen bulb of $150 \mathrm{~W}$ is used. Luminescence of the irradiated crystal is detected by a monochromator with the photon - counting photomultiplier system. Desorbed atom flux signal and the luminescence intensity could be recorded simultaneously as a function of time.

\section{Results}

The dependence of the $\mathrm{Rb}$ and I desorption yield on the time of irradiation (the electron dose) is presented in Fig. 1. The oscillations of the desorption yields are connected with the varying topography of the sample surface: the desorption coefficient increases with increasing num-

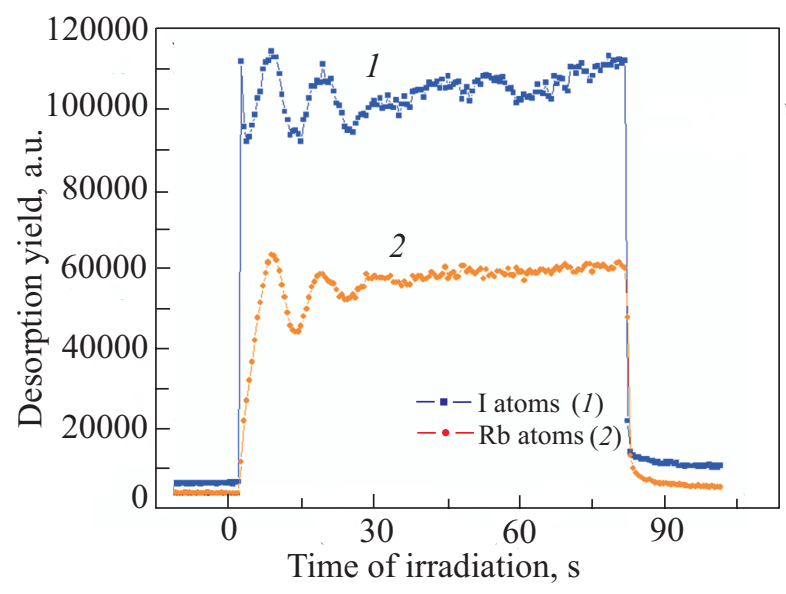

Fig. 1. (Color online) The electron stimulated desorption signal of $\mathrm{Rb}$ and $\mathrm{I}$ atoms as a function of the irradiation time (the electron dose), measured at sample temperature of $370 \mathrm{~K}$. The electron beam of spot size of about $1 \mathrm{~mm}^{2}$ was scanned over the area of $1.5 \mathrm{~cm}^{2}$ (electron density $\sim 3.3 \mu \mathrm{C} / \mathrm{cm}^{2}$ per second). 
ber of surface steps and edges, since they act as traps for diffusing defects and enable the F-centers to cause ejection of an alkali atoms. The desorption yield reaches its maximum when half of the monolayer is removed. Then, the desorption signal decreases and the minimum of the desorption process is observed when the whole upper monolayer is being removed. After a few periods of oscillations, the process does not follow the layer-by-layer mode anymore, and the oscillations are damped. The connection between the desorption yield changes and the surface topography has been confirmed previously by the AFM measurements $[11,12]$.

In Fig. 2 the changes in partial desorption yields caused by co-excitation, with the electron beam and visible light (photons of energies about 2-2.5 eV), are presented for both, $\mathrm{Rb}$ and I atoms. Since the measurement is performed with the electron beam irradiating a single spot (contrary to the case shown in Fig. 1, where the beam is scanned over the sample surface), there is no the oscillatory behavior of the desorption signals due to fast erosion of the surface topography averaging the number of the desorption sites (for more explanation see Discussion section). It is clear that additional illumination of the sample with light causes significant increase of the desorption yield for both halogen and alkali atoms.

In further steps of our work mass selected time-of-flight (TOF) spectroscopy is used to study velocity distributions of the desorbing atoms. It has been demonstrated in many experiments [20-22], that the TOF spectra of halogen atoms consist of two components: the thermal (which can be fitted with a Maxwellian distribution function) and the non-thermal one. The non-thermal peak, at energy of about $0.25 \mathrm{eV}$, corresponds to the halogen atoms emitted as a result of the decay of self-trapped excitons at the surface. This component is temperature-independent, and does not exhibit the oscillatory behavior on the irradiation time.
Atoms desorbed in that process have anisotropic angular distribution, so that they are ejected preferentially along the $<100>$ axis. It has been already shown that such hyperthermal halogen emission occurs for most of the alkali halide crystals, except the sodium compounds [20]. The comparison of time-of-flight spectra for I atoms emitted from electron bombarded RbI sample with and without simultaneous white light co-excitation at 4 different temperatures of the sample is shown in Fig. 3. The pronounced increase of the yield of thermally desorbed atoms is clearly visible, whereas the non-thermal peak remains unchanged at all investigated temperatures.

The corresponding TOF spectra of rubidium atoms desorbed by electron beam with and without light illumination are shown in Fig. 4. As already established in our previous reports there is no the non-thermal component for alkali atoms. Although it is already shown that the simultaneous illumination with the white light photons causes the increase in the total emission of $\mathrm{Rb}$ atoms (the QMS signal is enhanced as shown in Fig. 2), the Maxwellian peak in the TOF spectrum taken for both temperatures has lower intensity for the experiment with light co-excitation than for the electron bombardment alone. As it is described in the section Discussion, the excited F-centers require some time to diffuse to the surface and cause the desorption. The timescale of their diffusion, which depends also on the sample temperature, is much longer than $1 \mathrm{~ms}$ (time of the single TOF measurement), that is why most atoms, emitted as a result of a single electron pulse, are detected after some considerable delay time, and they are not shown in the TOF spectrum displayed in Fig. 4. There is also some excitation and emission occurring between the electron pulses, since the sample is light irradiated constantly, not only during the electron bombardment. The delayed thermal peak is also not exactly the Maxwellian distribution, what is clearly seen for lower sample temperatures.
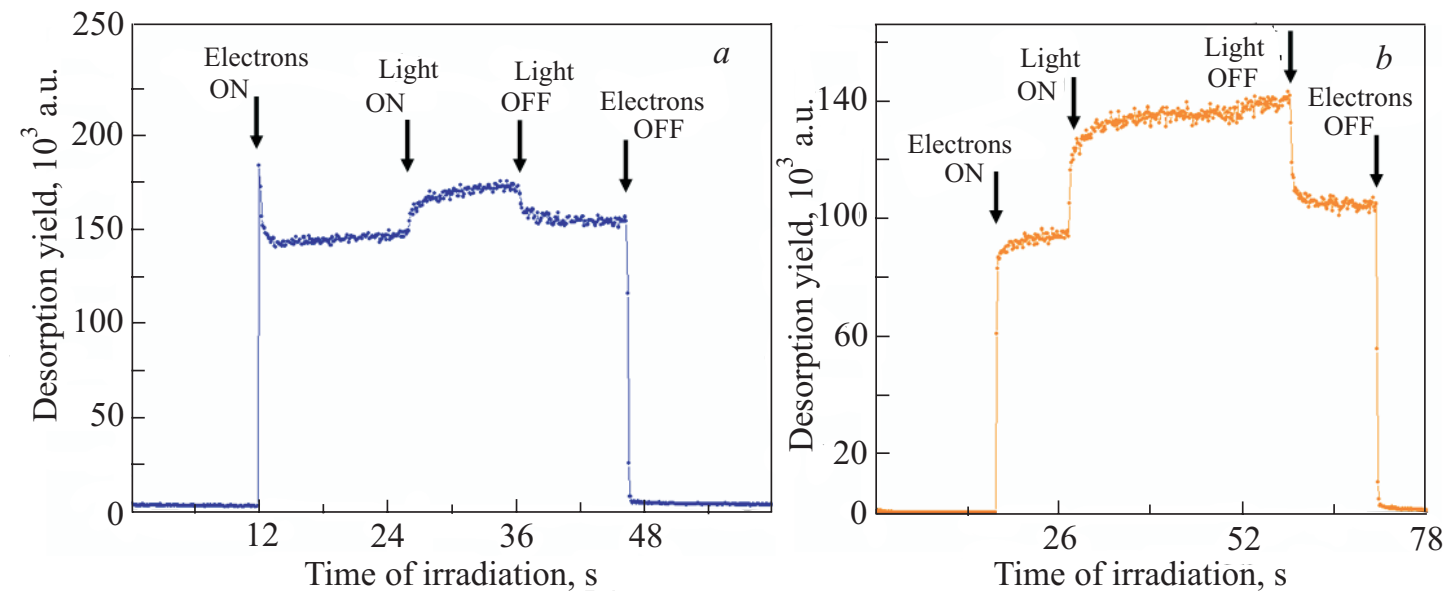

Fig. 2. Changes of $\mathrm{I}(a)$ and $\mathrm{Rb}(b)$ quadrupole mass spectrometer signals for electron irradiated $\mathrm{RbI}(001)$ surface with and without simultaneous co-excitation of the F-centers with visible light. 

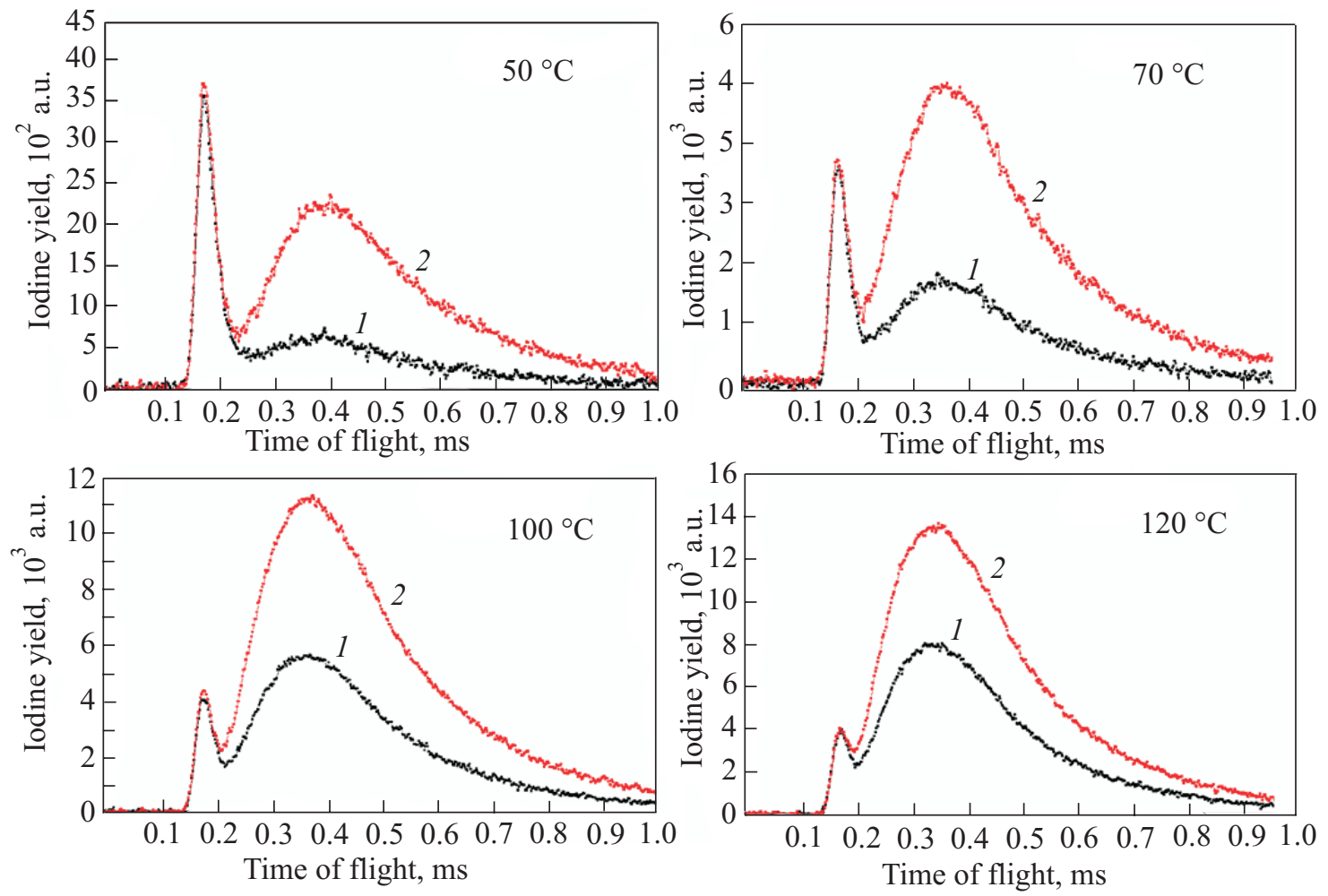

Fig. 3. (Color online) Time-of-flight spectra of iodine atoms emitted from RbI surface irradiated with $1 \mathrm{keV}$ electrons, measured for various sample temperatures. The black curves (1) present the TOF spectra obtained without the simultaneous illumination with visible light whereas the red curves (2) are the corresponding TOF spectra obtained with simultaneous white light illumination of the electron irradiated sample. All spectra are recorded for observation angle 0 degrees (along normal to the surface plane). The electron beam incidence angle is $\sim 45^{\circ}$.

\section{Discussion}

It has been established that the electron-induced desorption yield of the alkali halide crystal depends, firstly, on the topography of the surface and, secondly, on the concentration of Frenkel defects in the subsurface region of the electron irradiated crystals $[9,18]$. As for the first effect, the influence of the surface topography on the efficiency of ESD process, the following scenario has been proposed to explain the oscillatory behavior of the desorption signal on the irradiation time seen in Fig. 1. Electron bombardment of the crystal leads to production of the pairs of defects, i.e., mobile excited $\mathrm{F}^{*}$-centers (electrons trapped in the halogen vacancies) and H-centers (halogen interstitial atoms). These defects diffuse independently inside the crystal. When the H-center reaches the surface, the halogen atom is promptly emitted. On the other hand, excited $\mathrm{F}^{*}$-centers recombining with the surface, induce
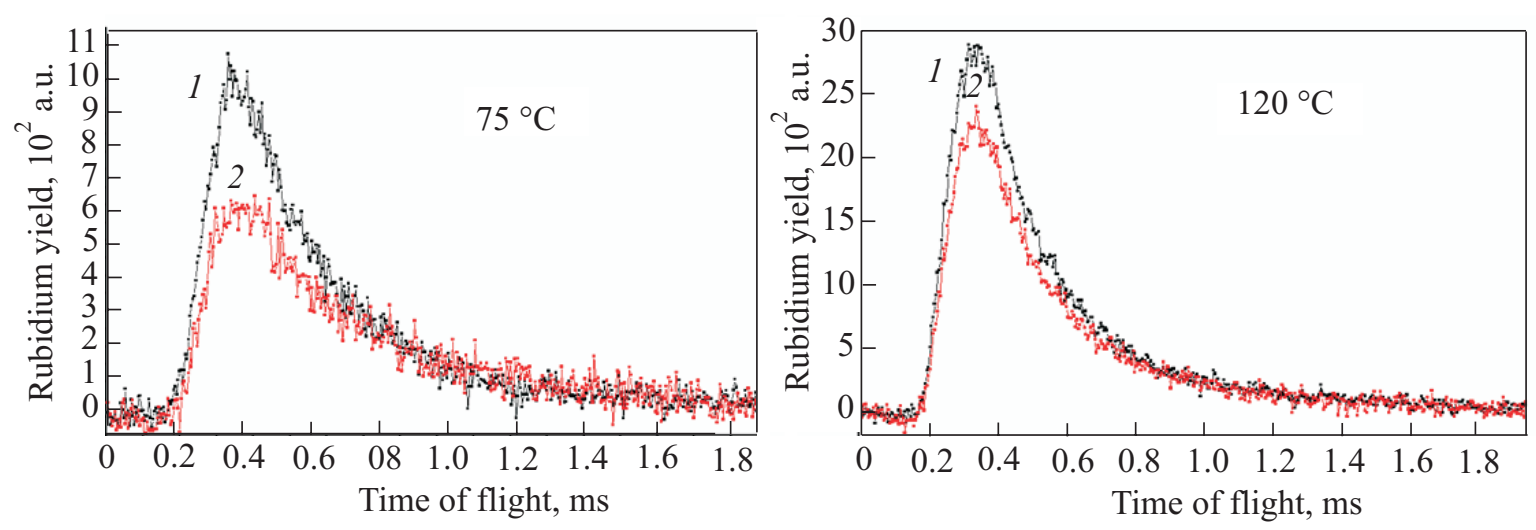

Fig. 4. (Color online) TOF spectra of rubidium atoms emitted from $\mathrm{RbI}(001)$ surface irradiated with $1 \mathrm{keV}$ electrons, measured for two different sample temperatures. The black curves (1) present the TOF spectra obtained without the simultaneous illumination with visible light whereas the red curves (2) are the corresponding TOF spectra obtained with the simultaneous light illumination of the electron irradiated samples. The delay in atom emission decreases with increasing temperature. 
emission of the alkali atoms, and consequently lead to formation of molecular vacancies on the surface. Furthermore, it has been shown by theoretical calculations [10] that the excited $\mathrm{F}^{*}$ - center cannot initiate desorption unless it is trapped at low coordinated sites (like terrace edges, kinks). Thus, at the surface, depending on its roughness, different fractions of excited $\mathrm{F}^{*}$-centers are reflected back to the bulk where they de-excite and stabilize. The steadystate concentration of immobile F-centers in the subsurface region is built up after a certain time of irradiation. This concentration is controlled by the surface recombination/reflection rate for the diffusing $\mathrm{F}^{*}$-centers, which in turn depends on the surface step density. Since the desorption occurs in the layer-by-layer mode, oscillating surface step density leads to the observed oscillatory behavior of the desorbed flux of alkali atoms (see Fig. 1). Varying concentration of immobile F-centers at the surface proxymity modulates probability of the $\mathrm{H}-\mathrm{F}$ annihilation what eventually results in yield modulation of the complementary desorption process of the halogen component. Consequently, the emission of both constitutes of the alkali halide crystal is influenced by the surface topography.

As for the second effect, the role of concentration of Frenkel defects, it turns out that the desorption efficiency depends on the mobility and lifetime of those defects in the bulk. Therefore, changing F-center properties in the bulk should lead to changes of the desorption yield. Such an experiment with simultaneous irradiation of $\mathrm{RbI}(001)$ sample with electrons and white light is reported in this work in Figs. 2-4. For the co-excitation with light, a noticeable increase of the desorption yield for both sample constituents is observed. This can be explained by assuming that the ground-state F-centers, accumulated in the bulk due to electron irradiation, are very efficiently excited by a white light to their $2 p$-electronic state and become very mobile, so they escape very fast via desorption process from the surface (contributing to the increase of alkali component of desorption yield) instead of staying in the bulk and constituting traps for migrating H-centres. Thus, by F-center excitation with light, the rate of $\mathrm{F}-\mathrm{H}$ recombination process in the bulk is decreased. Since the F-H recombination in the bulk is a competing process to the $\mathrm{H}$ centre diffusion to the surface, the decrease of its efficiency results in enhancement of the desorption yield of the halogen thermal component.

This interesting effect of $\mathrm{F}-\mathrm{H}$ center bulk recombination on the desorption yield of surface constituents is further supported by investigations of the $\pi$-luminescence intensity emitted by the desorbed sample. Since the desorption process should be competitive to the $\mathrm{F}-\mathrm{H}$ recombination in the bulk of the crystal, one would expect that phases of the oscillatory signal of desorption and luminescence are opposite. Results of the simultaneous measurements of the desorption yield and the luminescence intensity are shown in Fig. 5. Although the excitation of the accumulated F-centers leads to an increase in the desorption and luminescence signals but both signals are in phase. Feasible explanation of such a relationship requires that F-center participating in the radiative annihilation of the $\mathrm{F}-\mathrm{H}$ pair is in the electronically excited state. Nonradiative decay, in turn, occurs only if the F-center at the moment of recombination is in its ground state. Consequently, when the desorption signal passes through its maximum, the concentration of excited, mobile F-centers in the vicinity of the surface is high. At the minimum of the desorption signal majority of beam generated F-centers is reflected from the relatively undefected surface [11] accumulating in the bulk as immobile, ground state defects. Consequently, the recombination of $\mathrm{H}$-centers with the accumulated ground state F-centers is nonradiative.

\section{Conclusions}

Mass selected time-of-flight spectroscopy has been used for studying desorption processes occurring on RbI (100) surfaces due to simultaneous electron and white light coexcitation. Our results demonstrate that F-centers play a crucial role in the ESD process. Although the ground-state F-centers are immobile, theirs excitation either by the primary beam itself, or by illumination with light within the absorption F-band, results in efficient transport of the excited defects from the bulk to the surface and detailed balancing of the thermal desorption component of halogen versus alkali atoms. Therefore, simultaneous irradiation of the crystal by electrons and visible light within the F-center absorption band increases average F-center mobility, and therefore, has a profound effect on both the morphology of the surface and the desorption process efficiency. Such processes could be applied to increase efficiency and precision of the controlled surface nanostructuring of ionic

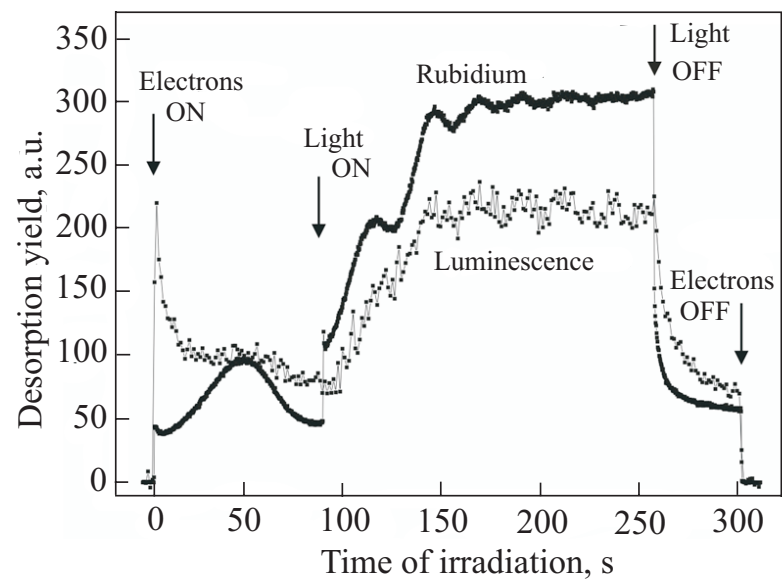

Fig. 5. Dependence of Rb desorption signal and the corresponding $\pi$-luminescence on time of electron irradiation for $\mathrm{RbI}(001)$ surface with simultaneous co-excitation with the white light. The moments of turning on and off of the light are indicated by arrows. Electron beam energy is $1 \mathrm{keV}$ and the sample temperature is of $100{ }^{\circ} \mathrm{C}$. 
insulators. In contrast, prompt emission of non-thermal halogen atoms due to electronic excitations with energy higher than the bandgap energy, and surface recombination of the excited electron-hole pairs, is not affected by additional illumination with the white light.

1. For recent reviews see: N. Itoh and A.M. Stoneham, Materials Modification by Electronic Excitation, Cambridge University Press (2001); E. Gnecco and M. Szymonski, Nanoscale Processes on Insulating Surfaces, World Scientific Publishing Co., Singapore (2009).

2. M. Szymonski, Radiat. Eff. 52, 9 (1980).

3. W.P. Hess, A.G. Joly, K.M. Beck, V.P. Sushko, and A.L. Shluger, Surf. Sci. 564, 62 (2004).

4. E.M. Williams and R.E. Palmer, Desorption Induced by Electronic Transitions, Elsevier, New York (1997).

5. E.V. Savchenko, G.B. Gumenchuk, I.V. Khyzhniy, A.N. Ponomaryov, and V.E. Bondybey, Surf. Sci. 6023204 (2008).

6. M. Goryl, FB de Mongeot, F. Krok, A. Vevecka-Priftaj, and M. Szymonski, Phys. Rev. B76, 075423 (2007).

7. L. Nony, E. Gnecco, A. Baratoff, A. Alkauskas, R. Bennewitz, O. Pfeiffer, S. Maier, A. Wetzel, E. Meyer, and C. Gerber, Nano Lett. 4, 2185 (2004).

8. L. Nony, R. Bennewitz, O. Pfeiffer, E. Gnecco, A. Baratoff, E. Meyer, T. Eguchi, A. Gourdon, and C. Joachim, Nanotechnology 15, S91 (2004).

9. M. Goryl, B. Such, F. Krok, K. Meisel, J.J. Kolodziej, and M. Szymonski, Surf. Sci. 593, 147 (2005).

10. V. Puchin, A. Shluger, Y. Nakai, and N. Itoh, Phys. Rev. B49, 11364 (1994).
11. B. Such, J. Kolodziej, P. Czuba, P. Piatkowski, P. Struski, F. Krok, and M. Szymonski, Phys. Rev. Lett. 85, 2621 (2000).

12. B. Such, P. Czuba, P. Piatkowski, and M. Szymonski, Surf. Sci. 451, 203 (2000).

13. H. Hoeche, J.P. Toennies, and R. Vollmer, Phys. Rev. B50, 679 (1994).

14. M. Szymonski, A. Droba, M. Goryl, J.J. Kolodziej, and F. Krok, J. Phys.: Condens. Matter 18, S1547 (2006).

15. F. Krok, J.J. Kolodziej, B. Such, P. Piatkowski, and M. Szymonski, Nucl. Instr. Meth. B212, 264 (2003).

16. T. Kubo, A. Okano, J. Kanasaki, K. Ishikawa, Y. Nakai, and N. Itoh, Phys. Rev. B49, 4931 (1994).

17. J. Kolodziej, B. Such, P. Czuba, F. Krok P. Piatkowski, P. Struski, M. Szymonski, R. Bennewitz, S. Schar, and E. Meyer, Surf. Sci. 482-485, 903 (2001).

18. M. Szymonski, J. Kolodziej, B. Such, P. Piatkowski, P. Struski, P. Czuba, and F. Krok, Prog. Surf. Sci. 67, 203 (2001).

19. M. Szymonski, P. Struski, A. Siegel, J.J. Kolodziej, B. Such, P. Piatkowski, P. Czuba, and F. Krok, Acta Phys. Polonica B33, 2237 (2002).

20. A. Droba, Ph. D. Thesis, Jagiellonian University, Krakow (2006).

21. J. Kolodziej, Z. Postawa, P. Czuba, P. Piatkowski, and M. Szymonski, Radiat. Eff. 128, 47 (1994).

22. M. Szymonski, J. Kolodziej, P. Czuba, P. Piatkowski, and A. Poradzisz, Phys. Rev. Let. 67, 1906 (1991).

23. A. Alexandrov, M. Piacentini, R.G. Tonkyn, M.T. Sieger, N. Zema, and T.M. Orlando, Surf. Sci. 451, 208 (2000). 